\title{
EXPLORACIONES EN LA SIERRA DE JUAREZ
}

\author{
Por el Dr. Ladislao PARAY
}

En el mes de abril emprendimos un viaje de dos semanas atraves de de la Sierra Madre Oriental llamada la Sierra de Juárez. Comúnmente refiere a dicha sierra como la de Juórez pero, en realidad, geográficamente se trata de la Sierra Madre oriental. Esta región apartada y de dificil acceso, se divide en varias partes. Sierra de Juárez se llama la región alrededor de Ixtlán de Juárez, Natividad etc. De Talea de Castro se llama la Sierra de San Nolasco. De Talea a Villa Aita recibe el nombre de Sierra del Distrito de Villa Alta. Adelante de Villa Alta a Choapán y más abajo hacia la costa es la del Distrito de Choapan. También se conoce la región con el nombre de La Chinantla. Los habitantes de la región son indígenas que pertenecen a los grupos étnicos de los chinantecas, los zapotecas y los mijes.

Empezamos nuestro recorrido en el mineral de Natividad, recorriendo la región de Talea de Castro,Playa Vicente Ver. Es difícil imaginar una región más accidentada y más salvaje que este rincón de la Re. público Mexicana. En todars partes se elevan montañas, separadas por profundas barrancas. Las distancias entre los escasos pueblos en línea recta no son importantes, pero por tierra son enormes, debido a lo anfractuoso del terreno. Para llegar de un pueblo al otro hay que bajar mil metros por la ladera de una montaña y subir otros mil en el otro lado. Hay que atravesar caudalosos ríos donde no existen puentes o en el mejor de los casos hay hamacas o puentes colgantes en estado deteriorado y por lo tanto peligroso. Las montañas están cubiertas de espesos bosques semitropicales donde es necesario abrir brecha a $m a$ chetazos: Las poblaciones son escasas y a veces se necesita caminar treinta o cuarenta $\mathrm{Km}$. para encontrar alguna ranchería. Existen lugares como Chopan que están a más de cien $\mathrm{Km}$. de la más cercana carretera. Las alturas que recorre uno en una jornada varían entre los mil y tres mil metros y en la última etapa se llega casi al nivel del mar (Playa Vicente, Ver.) Todas estas circunstancias hacen de esta región una de las más interesantes y más pintorescas del país y su ve-

Paray L. 1951. Exploraciones en la Sierra de Juárez. Boletín de la Sociedad Botánica de México 13: 4-10. 
getación es de las más variadas curiosas .

Debido a las enormes dificultades que hay que vencer para penetrar en una región tan hosca y tan inaccesible, pocos han sido los exploradores que se han aventurado en estas majestuosas montañas. En el siglo pasado dos batánicos daneses; Liebmann y Oersted colectaron en esta región, y en la época actual los Dres. Reko y Schultes colectaron en algunas partes de esta inmensa serranía. En general podemos decir que la región está poco explorada y todavía encierra muchas especies nuevas o poco conocidas y pasarán muchos años antes de que los exploradores botánicos lleguen a ciertos lugares de esta abrupta sierra, adonde hoy es poco menos que imposible llegar. En un reciorrido de esta índole uno trapieza con las plantas más variadas, desde el pino de las elevadas alturas hasta los vegetales de los climas tropicales.

Esta parte del Estado de Oaxaca se caracteriza especialmente por el gran número de especies de Q:ercus, Ericáceas, Lauráceas, y del género Clethrar. Pero sobre todo predominan las Ericáceas (géneros; Xolisma, Arctostaphylos Beffaria Gaultheria, Vaccinium, etc.)

Iniciando nuestro recorrido por la sierro de San Pedro Nolasco subimos en un alto cerro llamado de la Maceta. La vegetación en este cerro es todavía virgen y se puede afirmar que hasta ahora el hacha nunca ha llegado a este lugar. Espesos bosques cubren todo el macizo extenso del recio cerro. La altura media es de $2000 \mathrm{~m}$. en las laderas por donde pasamos. Aquí observo la gran cantidad de Quercus: Quercus Chinantlensis, Q. juergensenii, Q. sororia, Q. Leilomannil, Q. scitophylla y furfuracea. En estos seculares robles y encinos vegetan miles de epífitas pertenecientes a las más variadas familias. Predominan las cactáceas epifitas como Aporccactus Conzahii, y A. Alagelliforms, Epiphyllum caudatum con grandes flores rojas y blancas Las Vacciniáceas epífitas están representadas por las bellas y vistosas especies de Macleania insignis, Corvendishia acumincita, y $\mathbb{C}$. crassilolia. El límite más septentrional de estas Vaccineas epifítas es en esta región y no se extienden más al norte, en cambio abundan de aquí hacia el Sur, hasta los Andes, donde es su centro de distribución. Entre las Bromeliáceas observo la Aechmea mexicana y. Aechmea Irienastil, la Ipiccairnia microntha, Ia Tillandsia utriculata $\mathbf{y} \mathbb{T}$, vidacea.

Además de los encinos noto gran cantidad de otras especies arbóreas. Abundan las siguientes especies de Pinus; P. pseudostrobus oaxacana, $\mathbf{P}$. ayacahuite, Lawsonii, $\mathbf{P}$. michoacana, $\mathbf{P}$. oocarpa, $\mathbf{P}$. Pringlei, $\mathbf{P}$. teocote, P. Montezumae y P. Hartwegil. Encuentro varias especies de Sym plecos como S. coccinea, S. speciosa y S. juergensenil. Las Ericáceas ar- 
bóreas están representadas por Arbutus lanurina, Bellaria leavis, B. discalor y B. mexicana; además abunda Arclostaphylos polifolia. Crece aquí un frondoso Oreopanax salvinii. Encuentro también la Gillbertía juergenseni. Hay Garrya laurifolia y varias especies de Clethra, como la C. subveolens, C. mexicana, C. macrophylla y C. Ienaía. Veo varias especies de Melíosma. Las Dilleniáceas están también representadas por varias especies de Saurauia, la M. pseudopeduncularis, M. Nelsonil y M. Willde. moannil. Entre las Lauráceas distingo Lisisea Neesiana, Persea lloccosa y P. Leibmannii, Hufelandia mexicana y Ocotea effusa. Veo Styrax pilosug y $S_{i}$ glabrescens:

La vegetación arbustiva es en extremo variada. Colecto Rapanea juergensenil, Ardisia compressa, Rubus eriocorpus, R. philyrophyllum $\mathbf{~} \mathbf{R}$. Lielomannil. Hirtella americana. Abuundan los helechos arborescentes como Cyathea, Hemitelia y Alsophila. Encuentro la Davila Kunthï y la Hampea fomentosa. Nota dos especies de Hibiscus: $\mathrm{K}_{\text {. }}$ bifurcatus e H. cosfatus, con flores rojas.

En un Iugar llamado Llano Verde encontramos gran cantidad de Liquidambar Styracillua y un precioso Eupatorium arbustivo, el E. sordidum Less, var. alrorubens. Esta especie de Eupatorium es tal vez la más vistosa. Crece cerca de los arroyos y alcanza hasta de $3 \mathrm{~m}$. de altura. Sus flores moradas exhalan aroma. Mencionaré que la primera vez encontré esta especie de Eupatorium en el patio del Museo Etnográfico de Pátzcuaro Mich. En realidad la planta se presta mucho al cultivo por su vistosa apariencia. En este lugar encuentro tres especies de Cestrum: C. nitidum. C. fulvessens, C. lasciculatum. Más abajo enciuentro el Cestrum Bentha. mii. También cerca del arroyo crece la bella Fuchsia arborea. Hay aquí una Pinguicula con flores intensamente rojas. Mencionaré que el Liquildambar en esta región se llama "Yavito". En un lugar rocoso y húmedo en plena selva cerca de un arroyo encuentro una de las especies herbáceas más bonitas y más ráras. Se trata de la Trimezia lurida precîosa Iridácea que hasta ahora no se ha encontrado más que en este lugar que se llama Cueva de Cervantes, en plena Sierra de San Pedro Nolasco. Esta planta se parece mucho a la Trigridia pavonia, pero es más chica $y$ de un aspecto extraño. También en este lugar junto a la Trimezia enctientro una interesante Liliácer; la Echeandia macrophylla. Un poco más lejos hallo otra especie; lo Echeardia Iongiolia La vegetación herbácea está representada aquí por varias especies de Rondeletia: como la $\mathbb{R}$.buddleoides con flores blancas, la $\mathbf{R}$. villosa y la $\mathbb{R}$. juergensenil. Abundo la bella Liliácea Smallacina scilloidea, con flores blancas. Naturalmente en una región de bosques húmedos semitropicales no pueden laltar los representantes de la interesante familia de Melastomácears. Esta fami- 
Lia está representarda por un gran número de géneros y especies. En lo más húmedo de los bosques encontramos el Heterocentron roseum, Tibouchine scorbriuscula y T. schiedeana, Mouririo muelleri, Clidemia chinanilana, Miconia auricullaia, artenostachya y M. chrysoneura. La más típica Melastomácea que nos acompaña a todo lo largo del camino es la Conostegia superbar, un bello arbusto de unos dos m. de alto con flores blancas y fragantes.

Al bajar del cerro de la Maceta, tan rico en especies nos acercamos al pueblo de Talea de Castro. Aquí descubro un interesante encino; el Quercus oxjacana cuyo isotipo se colectó precisamente en este lugar o sea entre Tenatze y Talea. Además de esta especie hay Quercus ocolarelolia. En estos encinos como trepadora descubro la interesante Loganiácea; el Gelsemium sempervirens Esta planta, que por primera vez encuentro aquí, crece también en Estados Unidos en la costa del Atlántico.

De Natividad a Talea de Castro hicimos dos jornadas y dejamos atrás unos sesenta $\mathrm{Km}$. Talea que quiere decir en español "orilla de un patio" está poblada por la rcrza zapoteca, que es la más progresista de la región. Como prueba de la inteligencia y cultura de los habitantes de Talea mencionaré que todos los vecinos tienen on las afueras del pueblo una parcela de bosque. Es decir en esta parcela cultivan varias especies de árboles que utilizan para sus necesidades. La ventaja de este sistema es evidente, pues en esta forma todos los vecinos cortan árboles en su parcela y no destruyen los bosques. Ojclá que este sistema tan inteligente se generalizara en toda la República.

Por lo demás los alrededores de Tallea están materialmente cubiertos de arbustos de Xolisma aquamulesor en plena flor. Pooos arbustos hay más decorativos que esta especie de Kollisma. Con sus hojas elegantes siempre verdes y con sus flores blancas y olorosas que materialmente cubren el arbusto tienen un aspecto en extremo agradable. Las parcelas de bosque de los vecinos contienen además de la Xolisma, Beffaria discolor "Qvercus oajacana, Pinus Strobus chiapensis, Liquidambar Styrerciflua o "yavito", Clethra snaveolens, Conostegia superba, Arbulus laurina y Cupressus Lindleyi. Hablando de Cupressus mencionaré que cerca de Talea y Tanetze hay un cerro que está cubierto totalmente de Cupressus Lindleyi. Es el cerro de Cupressus por excelencia, aunque su nonbre en zapoteca sea otro.

Cerca de Talea y a las orillas del río San Juan encontramos Podocarpus y Canna lulea, además, Vaccinium leucanthum, y un precibso arbusto en plena flor: la Raliceurea Grileottiana.

Continuamos nuestro viaje hacia Villa Alta. Esta población se distin- 
gue bien desde Talea pero para llegar a ella se necesita una jornada larga de ocho horas, Talea está en las laderas de una alta serranía mientras Villa Alta está en las laderas de una serranía de enfrente. Así es que primero hay que bajar unos mil metros en el fondo de una inmensia barranca y luego subir por el otro lado otros mil metros. En el fondo de. la barranca corre un caudaloso río; el río Cajones. Pero antes atravesamos otro río; el de Santa Gertrudis. La única manera de atrovesar el río Cajones es por un fantástico y peligroso puente colgante o sea hamaca. Con muchas precauciones y con cierta angustia iniciamos la marcha por el endeble y tambaleante artefacto llamado irónicamente puente. Afortunadamente llegamos sin ningún incidente al otro lado. Aquí las laderas de los cerros están cubiertos por una vegetación xerofítica de tierra caliente. Estamos en una altura de mil metros. Distingo Acanhocereus, Cephaloso. reus, Enterolobium cvclocarpum, Bawhinia, Plumeria acutiolia, Cochlospermun vitifolum "Celtis iguanea, Psidium, Brysonima, Vermonia, Senecio, Hechtia, Petrea volubilis, Hyphis, Bryophyllum calicinum etc. Encuentro dos especies de Rhus: $\mathbf{R}$. juglandifelia y $\mathbf{R}$. potentillaefalia.

Antes de llegar a Villa Alta pasamos por un pueblo zapoteca llamado Lachirioag. Estamos a la vista de los pueblos de Temascalapa y Yatzona. Villa Alta es un pueblo simpático enclavado en plena sierra de Juárez. La vegetación en los alrededores es la misma que veníamos observando en el camino.

Los más interesante de nuestro viaje fué indudablemente el recorrido entre Villa Alta y Choapan. En estas dos jornadas atravesamos una región extraordinaria tanto de punto de vista de la Botánica como de la Geografía. Estamos en el corazón de la inmensa e intrincada sierra de Juórez en la Chinantla. En la primera etapa de la jornada iniciamos una gran bajada hasta el río Royaga. Atravesamos grandes bosques de Clethra. Aquí diré algo de las Orquídeas: Encontramos gran variedad de estas bellas epífitas en los Quercus, Clethras y otras especies. La cantidad de orquídeas es tal, que se nos antoja como orgía o como cascadas. Los árboles están materialmente inundados de orquídeas. Abunda especialmente el Oncidium tigrinum, pero encontramos además Oncidium inçurvum, $O_{n:}$ maculatum, $O$. rellexum y $O$. diaphanum. El género Odonthoglossum está representado por las especies 0 . Cervomiesi, 0 . ciľrosmum, 0 . nebulosum, $O$. macularum, $O$. cordarum, 0 . beloglossum y 0 . rubescens. Entre el género Eoidendrum distinguimos E. polybulbon, E. vilellinum, E. ptero.

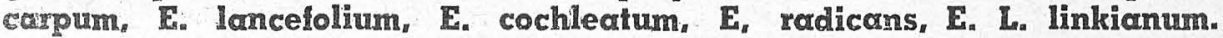
En los claros del bosque en encinos encontramos gran cantidad de Cemtleya chrina. La Stamnopea bucephalus se llama aquí "Yag-rabech" . Hay además Dichaea sonuarrose, Sobralia macrantha, Ornithidium 
densum. Lycaste aromática, Catasetum laminatum, Bletia adenocarpa, Alomania puricea, Galeandra Baueri, lonopsis paniculata, Arpophyllum giganteum. Todas estas especies de orquideas se encuentra de Natividad hasta mucho más abajo de Choapan, pero parece que donde más abundan es aquí empezando en el río Royaga y la cuesta de Tonaguía. Esta cuesta de Tonaguía es, por lo demás, lo más fantástico de todo el viaje.

Imagínense mis lectores una selva tupida semitropical nunca tocada por el hombre. Una estrecha brecha conduce através de los más tenebrosos bosques húmedos. Alrededor se ven oscuras montañas totalmente cubiertas por bosques nunca holladars por el pie humano. En los árboles centenares de monos arañas (Atteles) acampan y con una algarabía terrible reciben a los intrusos lanzándoles ramas y frutos. Esto es la selva virgen tropical tal como nos la imaginamos en nuestra juventud leyendo obras de aventuras truculentas. Naturalmente en un lugar como este podría pasar uno meses y años herborizando, pero unas cuantas horas son suficientes para darnos cuenta de la enorme riqueza vegetal. Uno de los árboles predominantes es el Quercus Tonaguiare, encino que lleva el nombre de esta localidad. Abundan laś Lauráceas, Simplocáceas; Trema micrantha, Ericáceas, Clethras, F'uchsia insermedia, Sarurauia, Siparuna nicaraguensis y riparia. Muchas de las plantas que colecté son tan raras que hasta la fecha no he podido clasificarless.

Tras de atravesar estos bosques vírgenes en una extensión de unos cuarenta $\mathrm{Km}$. llegamos a un pueblito olvidado en la selva que se llama Comaltepec. Este pueblo está en el fondo de una ancha barranca a las orillas del río del mismo nombre. Unas cuantas chozas, una escuela primitiva y una iglesia colonicl semidestruido forman ei pueblo.

El día siguiente reanudamos la marcha hacia Choapan a través de bosques de encinos en una altura de aproximadamente mil metros sobre el nivel del mar. Esta región es de la Melastomaceas. Además de la Conostegia superba encontramos Mịconia argentea, M. stenosiachya y $\mathbf{M}$. albicans. Abundan la Clidemia rubrar, que son plantas muy raras. Además abunda una rara Genciónacea que es el Chelonanthus, C. alatus o affine a ésta. Pero el hallazgo más raro fué un arbusto de aspecto elegante como de unos dos metros de altura. Sus flores en racimos colgantes nos recuerdan el Pensiemon. Me costó más de un año de búsqueda para identificarla. Se trata de la Uroskinnera hixtiflera Hemsl, de la familia de las Escrofulariáceass. En el Herbario Nacional existe un ejemplar con la anotación; "planta sumamante rora, se encuentra solamente cerca de Choapan (precisamente donde la encontré) existen únicamente cuatro ejemplares de ella en los distinios herbarios del mundo". Lo curioso es que el ejemplar del Herbario Nacional lo mismo que un dibujo de Hems- 
ley representan la planta con el racimo erecto, mientras yo las encontré todas con el racimo colgante, lo que precisamenfe caracteriza el arbusto que le da un ospecto extraño. Creo que el que la colectó primero puso el racimo de flores hacia arriba creyendo tal vez que esto era la postura natural de las flores. Nosotros encontramos el arbusto de Uroskinm nera siempre con los racimos colgantes y no hay duda de que esta es la colocación natural de las flores.

Cerca y en los alrededores de Chocrpan abundān las Palmas. Una die las más notables, es la Reinhard elea elegans que precisamente fué enlectoda aqui. Hay además Chomedorea ereophyla, Ch. liebmannil, Ch. humilis. De Choapan para abajo hacia la costa encontramos Astrocarm yum mexiconum, Desmoneus chinamtlensis, Scheelea Liebmanni: Ceonoma mexicana, Acrocomia mexicara y Sabal mexicana ya cerca del nivel del-mar. Saliendo de Choapan empieza la gran bajada hacia la costa Choapơn está a novecientos $\mathrm{m}$. sobre el nivel del mar. Sin embargo esta bajada no es rápida. Nosotros hicimos cuatro jornadas hasta Playa Vicente. A veces sube uno otra vez hasta $1500 \mathrm{~m}$., otrais veces baja uno hasta el fondo de profundas barrancas. Pasamos por el pueblo de Latani donde empieza la vegetación francamente tropical. La Cecropia pellata es llamada aquí "chancarro" la Pachira aquatica, "apompo" y donde abunda este árbol se llama apompales. Entre Latani y Jalahuy encontramos on bosques de encino una curiosa Solanácea; la Iuanulloa mexĭcana. Esta es una planta epifita con flores hermosas, tubulares y anaranjadas. Durante todo el día la vemos colgando de los enćinos. Parece que esta interesante planta se da solamente en esta región o cuando menos aquí es su centro de distribución.

En la región de Jalahuy abunda la Vainilla planifolla. Crece silvestre en los árboles. También encontramos una curiosa Passiflora con frutos comestibles que aquí se llama palau. Encontramos Pachira macrocarpa Helicteres guarumerelolia, Eugenia diebmannil, Trichilia haranensis, AIchome lartifolia, Pseudosmodinguin mulilolium, Acalpyha oligodonta Cissampelos parciro, Hollmannia reiulgens Vernonia deppeana, Lonchocarpus mexicanus, etc.

Pasando por las poblaciones de Boca del Monte. Xochiapa, llegamos al estado de Veracruz al nivel del mar. De Xochiapa pasando por Santa Cecilia iniciamos la marcha hacia Playa Vicente. En esta región abunda la Cepharlis tomentosa, Helicorior "Costus spicatus, Renealmia aromatica $Y$ especialmente una Melastomace de agradable perfume, la Mioonia ar gented.

En Playa Vicente Ver., terminó nuestro maravilloso viaje através de esta notable región del Estado de Oaxaca. 